strap pain $(46 \%)$ were the most prevalent symptoms. More than $10 \%$ of respondents rated experiences as "significant to severe" for blisters $(n=103)$, knee and ankle issues $(n=89)$, and sleep problems $(n=75)$. The most common environmental illness reported was altitude sickness $(37 \%)$. Hypothermia (7\%) and heat stroke $(5 \%)$ were uncommon. Constitutional complaints including headache (33\%), excessive fatigue $(41 \%)$, and excessive shortness of breath $(37 \%)$ were frequent. Falls were reported in $16 \%$ of hikers; $1.3 \%$ reported some form of fracture. Of respondents, $8.1 \%$ required medical attention, and $18.8 \%$ reported a medical problem that persisted for at least 2 weeks after the conclusion of their trips. Thirty hikers were forced to leave the trail for medical issues; 4 of these required emergency medical services assistance, including 3 helicopter evacuations.

Conclusions.-JMT hikers experienced medical issues similar to those reported on other national trails. Weight loss and altitude sickness were prevalent. A majority of hikers had medical complaints with a small percentage requiring medical attention.

\section{Technical Rescue Call Analysis of Roanoke County EMS for Creating WEMS Protocols}

Nathan Moore; Joshua Nichols; Stephanie Lareau

Virginia Tech Carilion, Roanoke, VA, USA

Introduction.--Roanoke County Emergency Medical Services (EMS) technical rescue team operates in southwest Virginia. Their operating area includes a variety of terrain, and they respond to approximately 30 technical rescue calls per year. The number of wilderness calls continues to increase as Roanoke becomes an outdoor destination. Roanoke County EMS does not have specialized wilderness protocols or standard equipment.

Objectives.-This study aims to evaluate the location, call type, and equipment used for Roanoke County technical search and rescue calls from 2014 to 2016 to develop standard equipment and protocols for wilderness rescue.

Methods.-Data for technical rescue calls between 2014 and 2016 were analyzed for global positioning system coordinates, call type (trauma, lost, animal injury), and whether patient transport was required. An analysis of standard equipment carried by team members was conducted.

Results.-From 2014 to 2016, Roanoke County EMS deployed on 90 technical rescue calls. A majority of calls took place at popular mountainous hiking areas along the Appalachian Trail, at either McAfee's Knob (38\%) or Dragon's Tooth (26\%), difficult locations to access by road. Based on the high percentage of incidents at these 2 locations, we further subdivided the data looking at Dragon's Tooth and McAfee's Knob. Of the total number of incidents $(n=59)$ occurring at McAfee's Knob and Dragon's Tooth, 40.6\% ( $n=24)$ involved trauma, 55.9\% $(\mathrm{n}=33)$ involved lost hikers, and $0 \%(\mathrm{n}=0)$ involved animal injury. Victim transport was required in $20.3 \%$ $(n=12)$ of calls. Response teams consisted of 7 to 10 EMS personnel, typically equipped with basic life support EMS kits.

Conclusions.-Most technical rescue calls for Roanoke County EMS take place at 2 popular locations along the
Appalachian trail, and many patients require transport. These data will be used to create standard wilderness equipment bags and protocols as well as training to more safely and efficiently respond to calls in our area.

\section{Apostle Islands National Lakeshore: a Review of Search and Rescue and Emergency Medical Services Operations, 2006-2015}

Elan Small ${ }^{1}$; Sarah Burbank ${ }^{1}$; Jeanette Lorme ${ }^{1}$; Karl Karlson ${ }^{2}$; Timothy Erickson ${ }^{3}$; David Young ${ }^{1}$

${ }^{1}$ Rush University, Chicago, IL, USA, ${ }^{2}$ Apostle Islands National Lakeshore, Bayfield, WI, USA, ${ }^{3}$ Brigham \& Women's Hospital, Boston, MA, USA

Objective.-Apostle Islands National Lakeshore (APIS) lies at the northern tip of Wisconsin and is home to a network of 21 islands along Lake Superior. The goal of this report is to investigate emergency medical services (EMS) and search-andrescue (SAR) trends at APIS in an effort to improve visitor safety and resource allocation.

Methods.-This study is a retrospective analysis reviewing APIS SAR Reports and Annual EMS Summary Reports from January 1, 2006 to December 31, 2015. Information related to incident type, incident time, individual demographics and activities, injury/illness type, cost, and contributing factors were recorded and analyzed in frequency tables. EMS analysis was limited to incident type.

Results.-From 2006 to 2015, APIS SAR conducted 133 total missions assisting 261 individuals: 57 injured/ill, 200 not injured/ill, 4 fatalities, and 25 documented saves. SAR incidents cost $\$ 3439$ on average, most frequently occurred during August and on Saturdays. Nonmotorized boating was the most common activity resulting in SAR incidents. Hypothermia/cold exposure accounted for over half of all injuries/illnesses $(45.0 \%)$. The most frequently reported contributing factor was wind. EMS responded to a total of 85 incidents with the majority of the EMS incidents involving first aid $(40.0 \%)$.

Conclusions.-Overall, this study highlights the hazards associated with the frigid, rough, and unpredictable conditions of Lake Superior. The results of this report will allow APIS personnel to more saliently convey risks to visitors in an effort to decrease the need for future rescues.

\section{Mushroom Poisoning}

William Brandenburg

Family Medicine Residency of Idaho, Boise, Idaho, USA

Objective.-To analyze mushroom poisoning epidemiologic data in the United States. Additionally, to review the types mushroom poisoning, causative organisms, and available treatments.

Methods.-Mushroom exposures and fatalities between 1999 and 2015 were obtained from the National Poison Data System. This information was compiled and statistically analyzed. Primary literature, textbooks, and field guides were 
used to review types of poisoning, culprit mushrooms, and available treatments.

Results.-Over the last 17 years 127,279 cases (7487/year) of mushroom exposure, mostly ingestion, have been reported. Cases are most frequently unintentional $(83 \%, P<.001)$, cause no or only minor harm $(86 \%, P<.001)$, occur in children $<6$ years old $(62 \%, P<.001)$. Approximately 665 (39/year) exposures have resulted in major harm. Fifty (2.9/year) fatalities have occurred, mostly $(83 \%)$ from cyclopeptide-containing mushrooms ingested by older adults (mean age $61.0 \pm 18.5$ years).

Conclusions.-The National Poison Data System recognizes 7 distinct groups of poisoning, although many more are likely to exist. The vast majority of ingestions result in no or minor harm, although some groups, like cyclopeptides, ibotenic acid, and monomethylhydrazine, can be deadly.

\section{Cardiovascular Implantable Electronic Devices and Scuba Diving: from Cardiac Electrophysiol- ogy Point of View}

Anthony Chang

Virginia Heart, Falls Church, VA, USA

Introduction.-People with cardiovascular implantable electronic devices (CIEDs) may wish to participate in scuba diving (SD). To best advise these patients, health care providers must have the necessary knowledge about CIEDs, including the impact of hyperbaric pressure, the indications, and the individual characteristics and limitations of different CIEDs.

Objective.-To review literature on CIEDs and SD and to present the latest information on CIEDs relating to hyperbaric pressure and the current CIEDs guidelines relevant to diving.

Methods.-The literature on CIEDs and SD, including technical documents of CIEDs from 4 major manufacturers (Biotronik, Boston Scientific, Medtronic, and St. Jude), and practice guidelines of CIEDs were reviewed. Testing methods, results, differential effects on the types of CIEDs (pacemaker, defibrillator, and loop recorder), and case reports were obtained from the manufacturers.

Results.-Existing literature on hyperbaric pressure and CIEDs contain outdated information. Recommendations regarding CIEDs and SD lack specifics on different types of CIEDs and do not address the underlying heart conditions. Indications for CIEDs range from mild symptoms to lifethreatening conditions that will limit SD. CIED test pressure varies from 3 to 7 ATM among manufacturers. Casing deformation can occur, but no component integrity or electronic function is compromised. Devices using piezoelectric sensors may cause undesired heart rate acceleration. No case of device malfunction related to diving or hyperbaric pressure has been reported to the manufacturers during the past 10 years. Some CIEDs are capable of wireless transmission using cellular and WiFi communicators best suited for remote monitoring.

Conclusions.- The underlying cardiac conditions should be the most important determinant when advising people with CIEDs who wish to engage in SD. Current generations of CIEDs function well under tested pressures. Knowing the individual characteristics and limitations of different CIEDs will help in selecting specific devices before implantation and in advising divers with existing devices.

\section{Death on the Dome: Epidemiology of Recrea- tional Deaths on Half Dome in Yosemite National Park}

Gregory Richardson; Susanne Spano

University of California San Francisco Fresno, Fresno, CA, USA

Introduction.-Half Dome is a popular destination in Yosemite with inconsistent public reporting of recreational mortality. The highest number of deaths in a single source is 20 .

Methods.-Multimedia sources describing deaths involving the cables, summit, climbing, or base jumping were included. Deaths occurring on trails were excluded.

Results.-Twenty-nine deaths occurred on Half Dome, with 2 additional deaths likely. The earliest was in 1930. The rate of 1 to 2 deaths per decade ended in the 1980 s, which saw 8 fatalities. The 2000s had 9 fatalities. This decade has 4 deaths currently. The typical profile is a 32-year-old hiking man (only 4 women were reported); the median age is 27 years (range 16-86). Hikers $(12 ; 39 \%)$ predominated, followed by 9 climbers $(29 \%)$. The most common mechanism was climbing incidents related to lost anchors (5), free climber falls (3), and head injury from rock fall (1). Fatal hiker falls from the cables (7) were occasionally weather related (3), and 71\% (5) occurred on descent. Lightning caused 3 summit deaths and a cablerelated death. Medical mortality, in 3 hikers, was attributed to cardiac causes (2) and altitude illness (1). There were 8 suicides (29\%). Base jumping fatalities (2) resulted from chute malfunction and wind slamming a jumper into Half Dome's face.

Conclusions.-We identified 31 Half Dome deaths, the highest published. A minority of deaths were attributable to unfavorable weather or unskilled hikers, despite this being a popular assertion. Climber accidents, often unpublicized, challenge the assumption that this increasingly popular sport is safe. These findings urge a particular educational focus on accidents during descent of the cables, a renewed focus on suicide prevention, and prompt consideration of permitting climber access to reduce mortality on Half Dome. 